EGU2020-18509

https://doi.org/10.5194/egusphere-egu2020-18509

EGU General Assembly 2020

(c) Author(s) 2020. This work is distributed under

the Creative Commons Attribution 4.0 License.

\title{
On the Rapid Spectral Evolution of Steep Wave Groups with Directional Spreading at Intermediate Depths
}

\author{
Dylan Barratt ${ }^{1}$, Harry B. Bingham², Paul H. Taylor ${ }^{1,3}$, Ton S. van den Bremer ${ }^{1}$, and Thomas A. A. \\ Adcock $^{1}$ \\ ${ }^{1}$ University of Oxford, Department of Engineering Science, Oxford OX1 3PJ, United Kingdom (dylan.barratt@eng.ox.ac.uk) \\ ${ }^{2}$ Department of Mechanical Engineering, Technical University of Denmark (DTU), 2800 Lyngby, Denmark \\ ${ }^{3}$ Faculty of Engineering and Mathematical Sciences, University of Western Australia, Crawley WA 6009, Australia
}

We have performed numerical simulations of steep three-dimensional wave groups, formed by dispersive focusing, using the fully-nonlinear potential flow solver OceanWave3D. We find that third-order resonant interactions result in directional energy transfers to higher-wavenumber components, forming steep wave groups with augmented kinematics and a prolonged lifespan. If the wave group is initially narrow banded, quasi-degenerate interactions resembling the instability band of a regular wave train arise, characterised by unidirectional energy transfers and energy transfers along the resonance angle, $\pm 35.26^{\circ}$, of the Phillips 'figure-of-eight' loop. Spectral broadening due to the quasi-degenerate interactions eventually facilitates non-degenerate interactions, which dominate the spectral evolution of the wave group after focus. The nondegenerate interactions manifest primarily as a high-wavenumber sidelobe, which forms at an angle of $\pm 55^{\circ}$ to the spectral peak. We consider finite-depth effects in the range of deep to intermediate waters (5.592 $\left.\geq k_{p} d \geq 1.363\right)$, based on the characteristic wavenumber $\left(k_{p}\right)$ and the domain depth $(d)$, and find that all forms of spectral evolution are suppressed by depth. However, the quasi-degenerate interactions exhibit a greater sensitivity to depth, suggesting suppression of the modulation instability by the return current, consistent with previous studies. We also observe sensitivity to depth for $k_{p} d$ values commonly considered "deep", indicating that the length scales of the wave group and return current may be better indicators of dimensionless depth than the length scale of any individual wave component. The non-degenerate interactions appear to be depth resilient with persistent evidence of $a \pm 55^{\circ}$ spectral sidelobe at a depth of $k_{p} d=1.363$. Although the quasi-degenerate interactions are significantly suppressed by depth, the interactions do not entirely disappear for $k_{p} d=1.363$ and show signs of biasing towards oblique, rather than unidirectional, wave components at intermediate depths. The contraction of the wavenumber spectrum in the $k_{y}$-direction has also proved to be resilient to depth, suggesting that lateral expansion of the wave group and the "wall of water" effect of Gibbs \& Taylor (2005) may persist at intermediate depths. 